DOI: 10.1515/ep-2017-0004

\title{
Ginton A. Examining Different Types of Comparison Questions in a Field Study of CQT Polygraph Technique: Theoretical and Practical Implications, Journal of Investigative Psychology and Offender Profiling, 2017
}

Since 1947 when the comparison question was first integrated by John Reid into the test format it became the key target of anti-polygraph criticisers. Not surprisingly, the Comparison (control) Question Techniques also happens to be the test technique most frequently used by polygraph examiners. Reid's Probable Lie Control Question (PLCQ), later labelled as the Non Exclusive Control Question (NECQ) was later changed by Backster into the Exclusive Control Question (ECQ). While the debate which CQ-NECQ or ECQ - is better underway, Raskin introduced the Directed Lie Control Question (DLCQ) in the 1980s. Since then more CQ-related ideas have been developed.

Many field examiners feel that what makes the question effective and responsive regardless of the format, whether it is a DLCQ or a PLCQ, is the manner in which the comparison question is introduced to the examinee in the pretest, and Ginton's latest research supports this notion. 
To start with, the research format is highly unique, it was integrated into real life in an examination of ongoing arson cases that in an Air Force base for over a year. During the investigation period over 300 alleged suspects were polygraphed. Out of this number, a group of 21 examinees were given a unique test format. They were faced with a traditional three relevant CQT test formats to include a primary involvement relevant question: "Did you, in person, set fire to one or more warehouses in the base?" a secondary involvement question: "Were you involved in setting fire to one or more warehouses in the base?" and a knowledge question: "Do you know for sure who set fire to one or more warehouses in the base?". Adjacent to these relevant questions were three different comparison type questions: one regular probable lie question: "Have you ever damaged any public property?" A hypothetical question: "Assuming you have very good reasons and opportunity, would you damage any public property out of rage or for pure fun?" And a bizarre question: "Do you like blue in particular?" The bizarre colour question was introduced to the examinee in the following manner (quoted from the research):

Look Ron, I am about to ask you a question that might look a kind of bizarre to you. But believe me, it is a very important question, otherwise I would not waste my time asking it. So, think very carefully before you answer me,-Do you like the color of blue in particular?"

Now, regardless of the examinee's answer, the interview went on to discuss the "blue" issue for another few minutes, relating to his or her habits and personality traits, and by so doing increased the salience of this question.

In case the examinee answered that blue is his favorite color the examiner asked him whether he considered his attraction to blue to be abnormal or pathological in its nature.

The final phrasing of the question was in accordance with this conversation, aiming to get a no answer. Thus, either it was "Do you like the color of blue in particular?" or "Do you consider your attraction to blue to be extremely abnormal?" and eventually, the answer chosen by the examinees was always "no".

As defined by Ginton the "study aimed to compare the three kinds of comparison questions to see whether there are any differences between them with regards to the strength of physiological reactions that they induced in the examinees". So in every repetition the physiological responses of the three different types of comparison questions were compared to each other and a rank order of the reactions' relative strength was established by an overall clinical judgment. The question judged as producing the strongest reactions, received 1 point, the second -2 points, and the weakest being in the third position received 3 points. All the charts were judged by three examiners separately. Later the number of points assigned by the judges to each 
question was totalled. The probable lie comparison question received a mean total of 1.94, the hypothetical comparison question -2.14 and the bizarre comparison question 1.91 , i.e. results that are remote from being statistically significant.

Ginton's research conclusion suggests that:

at least with truthful examinees, comparison questions, which do not incorporate any lies to be afraid of their exposure, or any lies at all, might function similarly to probable lie questions, by just increasing their salience in a manner that presumably creates some concerns about them.

The fact that the examinees' responses to three different comparison questions are similar led to the conclusion that Backster's "psychological set" concept that the innocent examinee's fear of detection assumingly causes the responses to the comparison question has no merit. Naturally Backster's supporters will disagree with this claim due to the fact that the comparison question was non-exclusive, a feature whose relevance was rejected in several studies.

Tuvya T. Amsel 